\title{
MicroRNA-761 inhibits the metastasis of gastric cancer by negatively regulating Ras and Rab interactor 1
}

\author{
QIFU ZHANG $^{1}$, YANA SUI ${ }^{2}$ and XIAOMEI SUI ${ }^{1}$ \\ ${ }^{1}$ Radiotherapy Department, Affiliated Hospital of Weifang Medical University; \\ ${ }^{2}$ Emergency Department, Weifang Traditional Chinese Hospital, \\ Weifang, Shandong 261000, P.R. China
}

Received October 5, 2018; Accepted May 17, 2019

DOI: $10.3892 / \mathrm{ol} .2019 .10645$

\begin{abstract}
Gastric cancer (GC), the second most common malignant cancer worldwide, gives rise to a number of cancer-associated fatalities annually. Accumulating evidence has shown that microRNAs (miRs) may serve as oncogenes or tumor suppressors in GC development. The aim of this study was to discover the expression, function and mechanism of miR-761 in GC progression. First, the findings revealed that the expression level of miR-761 was significantly decreased in GC cell lines and tissues. The functional studies showed that miR-761 in GC cells inhibited tumor proliferation and metastasis. In the mechanistic study, through an online database search and luciferase assay, Ras and Rab interactor 1 (RIN1), which has been demonstrated as an oncogene in various types of cancer, including GC, was identified as a target of miR-761. Notably, miR-761 expression was demonstrated to be negatively correlated with RIN1 mRNA levels in GC tissues. Simultaneously, overexpression of RIN1 partially rescued the inhibitory effect of miR-761 mimic in the GC cells. The present study provided new insights into the role of miR-761 in the progression of GC, and implicated the potential application of miR-761 in GC cell therapy.
\end{abstract}

\section{Introduction}

Gastric cancer (GC), the second most common malignant cancer worldwide, gives rise to a number of cancer-associated fatalities annually (1). Despite improvements in the diagnosis and treatment of the disease, however, the prognosis and the five-year survival rate of GC remains poor (2). Most patients with GC are diagnosed at an advanced stage, at which point GC treatment becomes difficult due to extensive tumor

Correspondence to: Dr Xiaomei Sui, Radiotherapy Department, Affiliated Hospital of Weifang Medical University, 465 Yuhe Road, Kuiwen Qu, Weifang, Shandong 261000, P.R. China

E-mail: suixiaomei2000@126.com

Key words: microRNA-761, gastric cancer, Ras and Rab interactor 1, proliferation, migration invasion and lymphatic metastasis $(3,4)$. Consequently, to provide improved GC interventions, a greater understanding of the mechanism of GC and accurate identification of novel therapeutic targets are necessary.

MicroRNAs (miRs/miRNAs) are a class of short non-coding RNAs of 22 nucleotides in length, with the ability to regulate gene expression by directing target mRNAs for degradation $(5,6)$. Several studies have shown that miRNAs are involved in regulating several cellular functions, including cell proliferation, apoptosis, motility and differentiation (6). In recent years, a great number of miRNAs have been identified and characterized as tumor suppressors or oncogenes in GC, and many of these molecules have been associated with GC metastasis, invasion and drug resistance $(7,8)$. miR-761 was recently reported to play important roles in human cancer, including breast cancer, hepatocellular carcinoma, non-small cell lung cancer, glioma and colorectal cancer (9-14). miR-761 serves as an oncogene or tumor suppressor in different types of cancer, depending on the tissue in which it is expressed. However, the expression, biological function and molecular mechanism of miR-761 in the progression of GC have not been well elucidated. Therefore, the determination of the function and mechanism of miR-761 in GC is essential.

In the present study, the expression level of miR-761 was decreased in GC tissues and cell lines compared with that in normal cells. Overexpression of miR-761 decreased, whereas knockdown of miR-761 promoted, the proliferation and migration of GC cells. The molecular mechanism study also demonstrated that RIN1 is a direct downstream target of miR-761 in GC cells, and that overexpression of RIN1 could rescue the inhibitory effect of the miR-761 mimic on the biological function of GC cells.

\section{Materials and methods}

Cell culture. Human GC cell lines MGC-803 (well differentiated adenocarcinoma), SGC-7901 (moderately differentiated adenocarcinoma), AGS (poorly differentiated adenocarcinoma) and BGC-823 (poorly differentiated adenocarcinoma), immortal human gastric epithelial GES-1 cells and 293T cells were obtained from the Cell Bank of the Chinese Academy of Sciences (Shanghai, China). All cells were grown in DMEM 
(Thermo Fisher Scientific, Inc.) containing 10\% fetal bovine serum (FBS; Thermo Fisher Scientific, Inc.) at $37^{\circ} \mathrm{C}$ under a humidified atmosphere containing $5 \% \mathrm{CO}_{2}$.

Human tissues. GC tumor tissues and adjacent normal tissues were obtained for the current study from 20 patients (age range, 49-71 years old; 12 males and 8 females) who underwent surgery for stomach cancer at the Affiliated Hospital of Weifang Medical University (Weifang, China) between June 2015 and June 2016. Prior written informed consent was obtained from each patient. Patients who underwent chemotherapy or radiotherapy prior to surgery were excluded from the current study. The study methodologies and the experiments were approved by the ethics committee of the Affiliated Hospital of Weifang Medical University (approval no. WYFY20150109002). Following removal, all tissues were stored at $-80^{\circ} \mathrm{C}$ immediately, until use.

Cell transfection. The miR-761 mimic (5'-UCAGCAGGC AGGCUGGUGCAGCGGTATTCGCACTGGATACGACGA ACTTT-3'), miRNA negative control (miR-NC; 5'-ACUACU GAGUGACAGUAGA-3'), miR-761 inhibitor (5'-GCUCCU GGAGCGUGUUAAUCCUGA-3') and inhibitor negative control (anti-NC; 5'-UUCUCCGAACGUGUCACGUTT-3') were obtained from Guangzhou RiboBio Co., Ltd. The open reading frame of RIN1 was inserted into pcDNA3.1 vector (Invitrogen; Thermo Fisher Scientific, Inc.) to generate the pcDNA3.1/RIN1 vector. The empty pcDNA3.1 vector (empty vector) was used as the control. A total of $5 \times 10^{5}$ SGC-7901 cells were transfected with miR-NC $(2.5 \mu \mathrm{g})$ or miR-761 mimic $(2.5 \mu \mathrm{g})$ and/or pcDNA3.1/RIN1 vector $(100 \mathrm{nM})$ or empty vector $(100 \mathrm{nM})$ using Lipofectamine ${ }^{\circledR} 2000$ reagent (Invitrogen; Thermo Fisher Scientific, Inc.) according to the manufacturer's protocol. A total of $5 \times 10^{5}$ BGC- 823 cells were transfected with anti-NC $(2.5 \mu \mathrm{g})$ or miR-761 inhibitor $(2.5 \mu \mathrm{g})$ using Lipofectamine ${ }^{\circledR} 2000$ reagent (Invitrogen; Thermo Fisher Scientific, Inc.) according to the manufacturer's protocol. Reverse transcription-quantitative PCR (RT-qPCR) was carried out to analyze the transfection efficiency after $48 \mathrm{~h}$. Western blot analysis was carried out $72 \mathrm{~h}$ after transfection.

Cell Counting Kit-8 (CCK-8) assay. A CCK-8 assay (Beyotime Institute of Biotechnology) was carried out to detect cell proliferation. GC cells $(3,000$ cells/well) were seeded into 96-well plates. Then, $10 \mu \mathrm{l} \mathrm{CCK-8} \mathrm{solution} \mathrm{was} \mathrm{added} \mathrm{to} \mathrm{the}$ wells. The absorbance of each well at $0,24,48$ and $72 \mathrm{~h}$ was detected at $450 \mathrm{~nm}$.

Colony-formation assay. Cells were transfected with miR-761 mimic, miR-NC, miR-761 inhibitor or anti-NC, as described above. The transfected cells were trypsinized, counted, and re-plated in a 6-well plate at a density of 400 cells/well. After 10 days, colonies resulting from the surviving cells were fixed with $4 \%$ paraformaldehyde for $10 \mathrm{~min}$ at room temperature, stained with $0.1 \%$ crystal violet for $5 \mathrm{~min}$ at room temperature and counted. Colonies containing $\geq 50$ cells were scored. Each assay was performed in triplicate.

Transwell assay. The migration and invasion assay was carried out using Transwell chambers (Corning Inc.). GC cells (5x104) were plated on the top chamber in DMEM (Thermo Fisher Scientific, Inc.) without serum. Medium containing 10\% FBS was added to the bottom chamber. Following incubation at $37^{\circ} \mathrm{C}$ in an atmosphere containing $5 \% \mathrm{CO}_{2}$ for $24 \mathrm{~h}$, cells on the lower filter were fixed with $4 \%$ paraformaldehyde (Beyotime Institute of Biotechnology) at room temperature for $20 \mathrm{~min}$, and stained with $0.5 \%$ crystal violet at room temperature for $10 \mathrm{~min}$. Cells migrating through the membrane were counted using a light microscope. For detecting cell invasion potential, $50 \mu 1$ pre-diluted Matrigel (BD Biosciences) was firstly added into the chambers and incubated at $37^{\circ} \mathrm{C}$ for $2 \mathrm{~h}$. The cells were later processed similar to that of cell migration assay.

Bioinformatics prediction. To investigate the possible target genes of miR-761, the online prediction system, TargetScan 7.1 software (http://www.targetscan.org), was used.

Dual-luciferase reporter assay. The wild-type (WT) or mutant (MUT) RIN1-3'UTR which contained the miR-761 binding sites was inserted into the psiCHECK2 vector (Promega Corporation). $293 \mathrm{~T}$ cells $\left(2 \times 10^{4}\right)$ were co-transfected with $0.1 \mathrm{mg}$ psiCHECK2-WT RIN1-3'-UTR or $0.1 \mathrm{mg}$ psiCHECK2-MUT RIN1-3'-UTR and $10 \mathrm{nM}$ miR-761 mimic or $10 \mathrm{nM}$ miR-761 inhibitor using Lipofectamine ${ }^{\circledR} 2000$ reagent (Invitrogen; Thermo Fisher Scientific, Inc.) according to the manufacturer's protocol. Cells were cultured $37^{\circ} \mathrm{C}$ for $24 \mathrm{~h}$ and luciferase activities were analyzed using the Dual-Luciferase Reporter Assay system (GeneCopoeia, Inc.) according to the manufacturer's protocol. The activity of firefly luciferase was normalized to the corresponding Renilla luciferase activity.

Western blot assay. Total proteins were extracted from cells using radioimmunoprecipitation lysis buffer (Thermo Fisher Scientific, Inc.) and the protein content was determined using the Bicinchoninic Acid Protein assay kit (Beyotime Institute of Biotechnology). Proteins $(30 \mu \mathrm{g})$ were separated by SDS-PAGE (10\% gels) and transferred to polyvinylidene fluoride membranes (EMD Millipore). Membranes were blocked at room temperature with 5\% non-fat milk for $1 \mathrm{~h}$ and incubated at $4^{\circ} \mathrm{C}$ overnight with the following antibodies: GAPDH (cat. no. ab9485; 1:500; Abcam), RIN1 (cat. no. ab251835; 1:200; Abcam), and subsequently with goat anti-rabbit secondary antibodies (cat. no. ab150077; 1:1,000; Abcam) at room temperature for $2 \mathrm{~h}$. The blots were detected by an enhanced chemiluminescence (ECL) system (Thermo Fisher Scientific, Inc.), and developed using X-ray film.

$R T-q P C R$. Total RNA was isolated from GC tumor tissues, adjacent normal tissues, GC cells and GES-1 cells using TRIzol reagent (Invitrogen; Thermo Fisher Scientific, Inc.) and cDNA was synthesized at $70^{\circ} \mathrm{C}$ for 5 min using a Maxima First Strand cDNA Synthesis kit (Thermo Fisher Scientific, Inc.), according to the manufacturer's protocol. The gene expression levels of RIN1 and miR-761 were determined using a SYBR Green qPCR kit (Thermo Fisher Scientific, Inc.), and calculated using the $2^{-\triangle \Delta C q}$ method (15). The PCR thermocycling conditions were: $94^{\circ} \mathrm{C}$ for 2 min followed by 30 cycles of $94^{\circ} \mathrm{C}$ for $30 \mathrm{sec}, 61^{\circ} \mathrm{C}$ for $30 \mathrm{sec}$, and $72^{\circ} \mathrm{C}$ for $30 \mathrm{sec}$. To determine RIN1 mRNA expression levels, GAPDH served as an internal control. To examine the expression levels of miR-761, U6 acted 

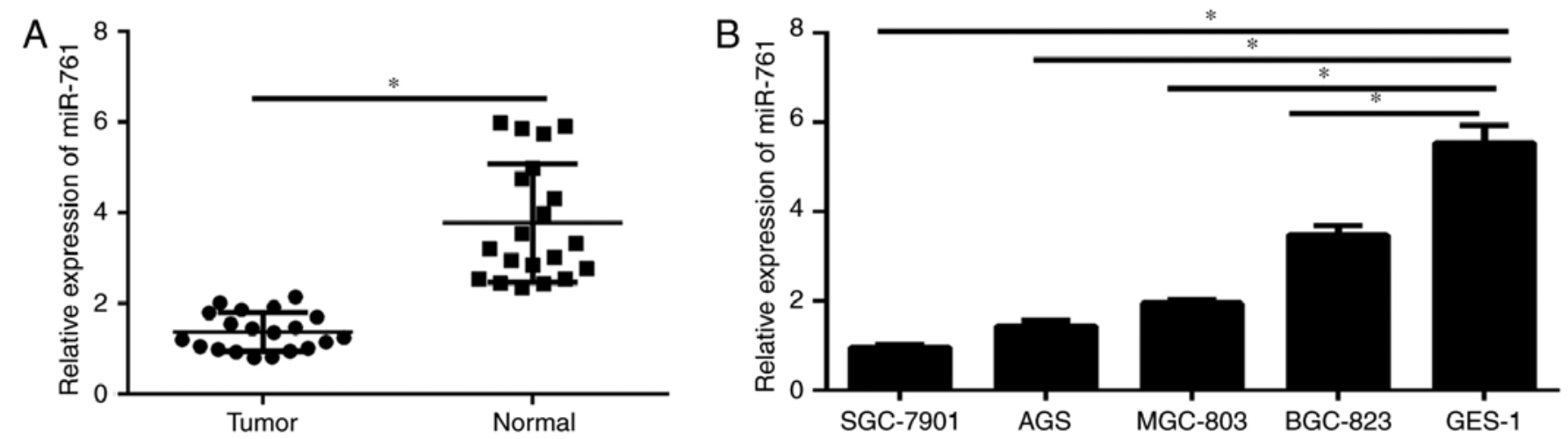

Figure 1. miR-761 is downregulated in GC tissues and cell lines. The expression level of miR-761 in (A) GC tissues and adjacent normal tissues, and (B) GC cell lines and GES-1 cells was analyzed using reverse transcription-quantitative PCR. U6 acted as the internal control. "P<0.05. miR, microRNA; GC, gastric cancer.

as the internal control. The primers used for amplification were as follows: miR-761 forward, 5'-ACAGCAGGCACAGAC-3'; miR-761 reverse, 5'-GAGCAGGCTGGAGAA-3'; U6 forward, 5'-CTCGCTTCGGCAGCACA-3'; U6 reverse, 5'-AACGCT TCACGAATTTGCGT-3'; RIN1 forward, 5'-GCACCTGGC GAGAGAAAAG-3'; RIN1 reverse 5'-TAGATTTCCGCACGA GGAACG-3'; GAPDH forward, 5'-GGTGATGCTGAGTA-3'; reverse 5'-GGATGCAGGGATGATGTTCT-3'.

Statistical analysis. Each experiment was repeated $\geq$ three times. Statistical analysis was carried out using SPSS software (version 13.0; SPSS, Inc.). Unless otherwise indicated, the data were evaluated as the mean \pm standard deviation. Pearson's correlation analysis was used to analyze the correlation between miR-761 and RIN1 mRNA expression. Data of $>$ two groups were analyzed using one-way analysis of variance with Tukey's post hoc test. Student's t-test was used to analyze the results between two groups. $\mathrm{P}<0.05$ was considered to indicate a statistically significant difference.

\section{Results}

miR-761 expression is downregulated in GC tissues and cell lines. RT-qPCR was carried out to analyze the expression level of miR-761 in GC tissues and cell lines. As demonstrated in Fig. 1A, the expression of miR-761 was significantly decreased in GC tissues compared with that in the corresponding normal adjacent tissues. In addition, miR-761 expression in GC cells and normal gastric epithelium GES-1 cells was analyzed. The results illustrated that miR-761 was significantly decreased in GC cell lines compared with that in the GES-1 cells $(\mathrm{P}<0.05$; Fig. 1B). These data indicate that miR-761 may play a critical role in the progression of GC.

miR-761 inhibits the proliferation of GC cells. To study the functional role of miR-761 in the progression of $\mathrm{GC}$, SGC-7901 and BGC-823 cells were selected for biological function experiments, based on the miR-761 expression results in the GC cells analyzed via RT-qPCR (relatively lower in the SGC-7901 cells and relatively higher in BGC-823). miR-761 mimic oligonucleotides were transfected into SGC-7901 cells and mir-761 inhibitor into BGC-823 cells, and its expression was detected via RT-qPCR. The results confirmed the transfection efficiency $(\mathrm{P}<0.05$; Fig. $2 \mathrm{~A})$. The results of the CCK- 8 assay showed that miR-761 overexpression decreased the proliferation of SGC-7901 cells transfected with miR-761 mimic compared with that of the cells transfected with miR-NC $(\mathrm{P}<0.05$; Fig. $2 \mathrm{~B})$, and the opposite effect was observed in BGC-823 cells subjected to miR-761 silencing ( $\mathrm{P}<0.05$; Fig. 2C). A colony-formation assay was performed to further examine the proliferation effects of miR-761. Consistent with the CCK-8 assay, the results of the colony-formation assay showed that miR-761 overexpression significantly decreased the colony-formation ability of SGC-7901 cells, whereas miR-761 inhibition exhibited the opposite effects in the BGC-823 cells $(\mathrm{P}<0.05$; Fig. 2D). These data suggest that miR-761 may act as a tumor suppressor in GC.

miR-761 inhibits the migration and invasion of GC cells. To further illustrate the function of miR-761 in the progression of GC, a Transwell migration and invasion assay was carried out. The results showed that the number of migrating and invading cells was significantly decreased in the SGC-7901 cells transfected with miR-761 mimic, and the opposite results were observed when miR-761 was silenced in the BGC-823 cells $(\mathrm{P}<0.05$; Fig. 3$)$. These data further indicate that miR-761 may serve as tumor suppressor in GC.

RIN1 is a direct downstream target of miR-761 in GC cells. The molecular mechanisms underlying the aforementioned functions were further explored. Among the potential candidates, detected via bioinformatic analysis (data not shown), RIN1 was selected as the focus of this study. The TargetScan target gene prediction analysis identified position 44-51 at the 3'untranslated region (3'-UTR) of RIN1 mRNA as a possible site of action of miR-761 (Fig. 4A). A luciferase reporter assay was performed to evaluate whether RIN1 is a direct target gene of miR-761. The results demonstrated that miR-761 markedly decreased the luciferase activity in the WT group compared with the miR-NC (Fig. 4B), whereas no significant differences were observed among the groups of cells with the Mut expression (Fig. 4B). Next, the results of the RT-qPCR and western blot assays showed 

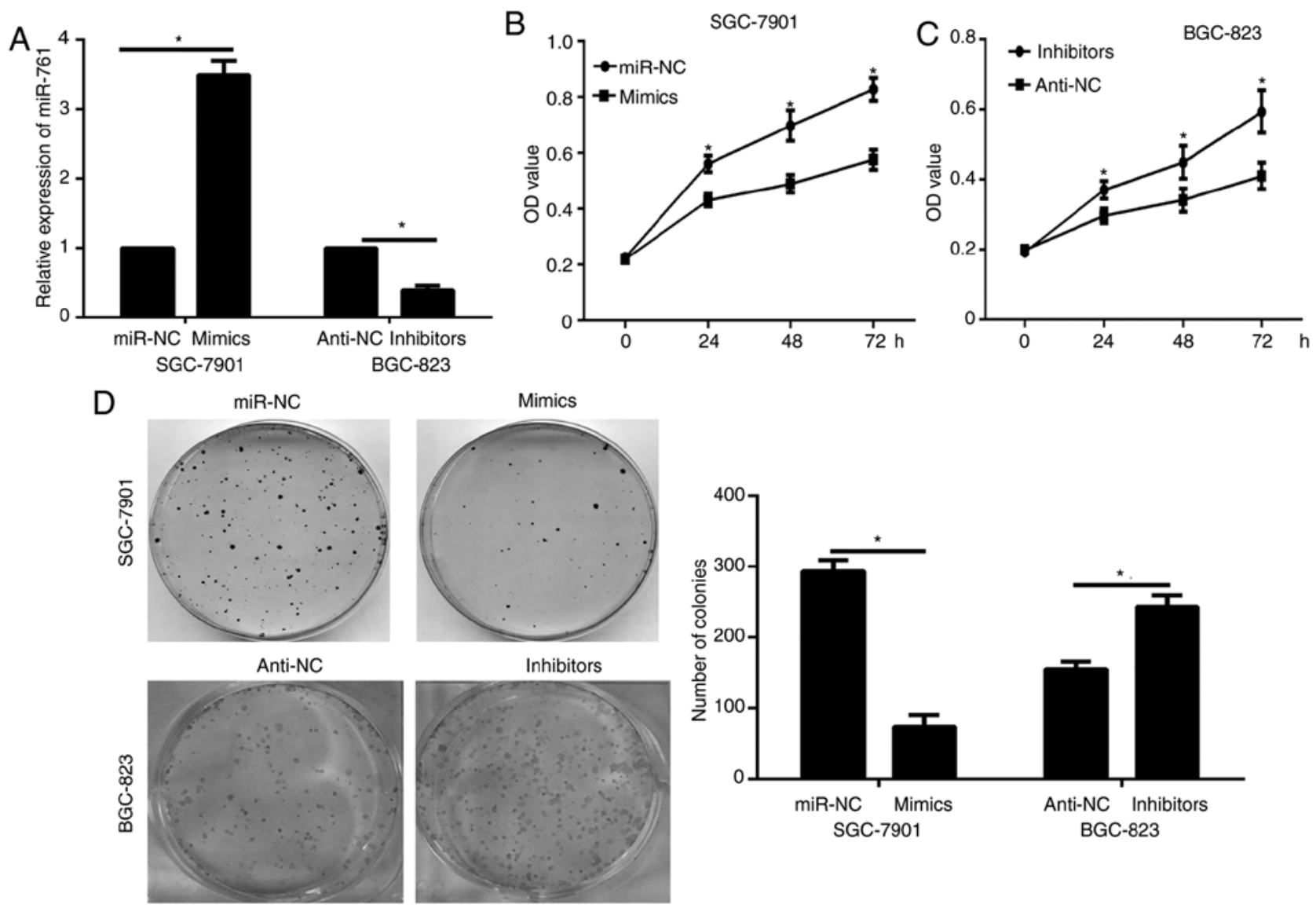

Figure 2. miR-761 inhibits the proliferation of gastric cancer cells. (A) The expression level of miR-761 in SGC-7901 (transfected with miR-761 mimic or $\mathrm{miR}-\mathrm{NC}$ ) and BGC-823 cells (transfected with miR-761 inhibitor and anti-NC), determined by reverse transcription-quantitative PCR. U6 acted as the internal control. The Cell Counting Kit-8 assay was used to detect the cell proliferation of (B) SGC-7901 and (C) BGC-823 cells. (D) A colony-formation assay was used to analyze the cell proliferation capability of SGC-7901 and BGC-823 cells. "P<0.05. miR, microRNA; miR-NC, microRNA negative control; anti-NC, inhibitor negative control.

A

Migration
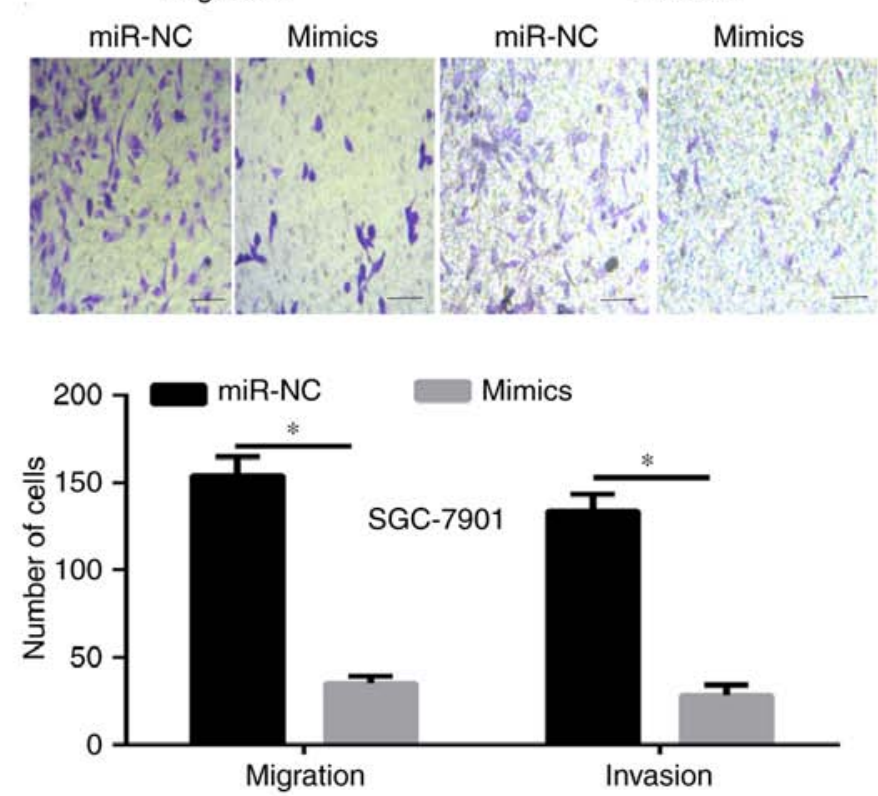

B
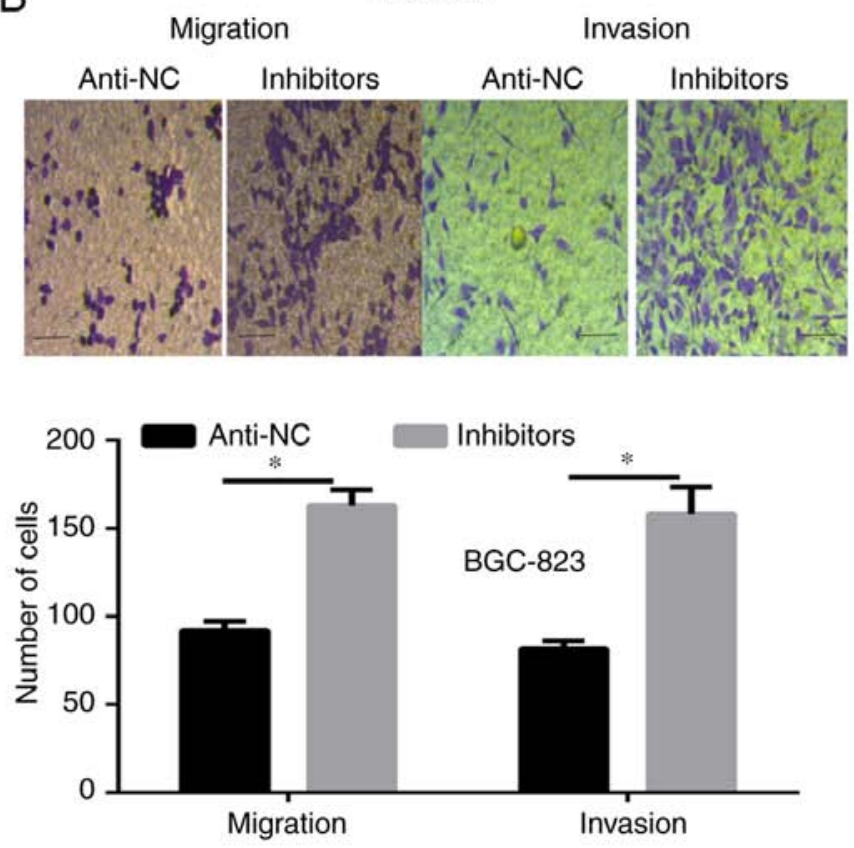

Figure 3. miR-761 decreases the migration and invasion of GC cells. The cell migration ability was assessed using a Transwell assay. (A) The ectopic expression of miR-761 in SGC-7901 cells decreased the migration and invasion capability. (B) The silencing of miR-761 in BGC-823 cells increased the migration and invasion capability. Scale bar, $100 \mu \mathrm{m}$. ${ }^{*} \mathrm{P}<0.05$. miR, microRNA; miR-NC, microRNA negative control; anti-NC, inhibitor negative control. 
A RIN1 WT $5^{\prime}$...GCGGGAGACCCUGAGCCUGCUGA...3'

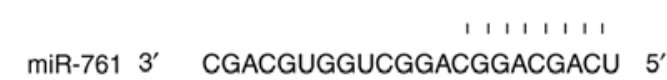

RIN1 MUT $5^{\prime}$...GCGGGAGACCCUGAGCGACGAGA... $3^{\prime}$

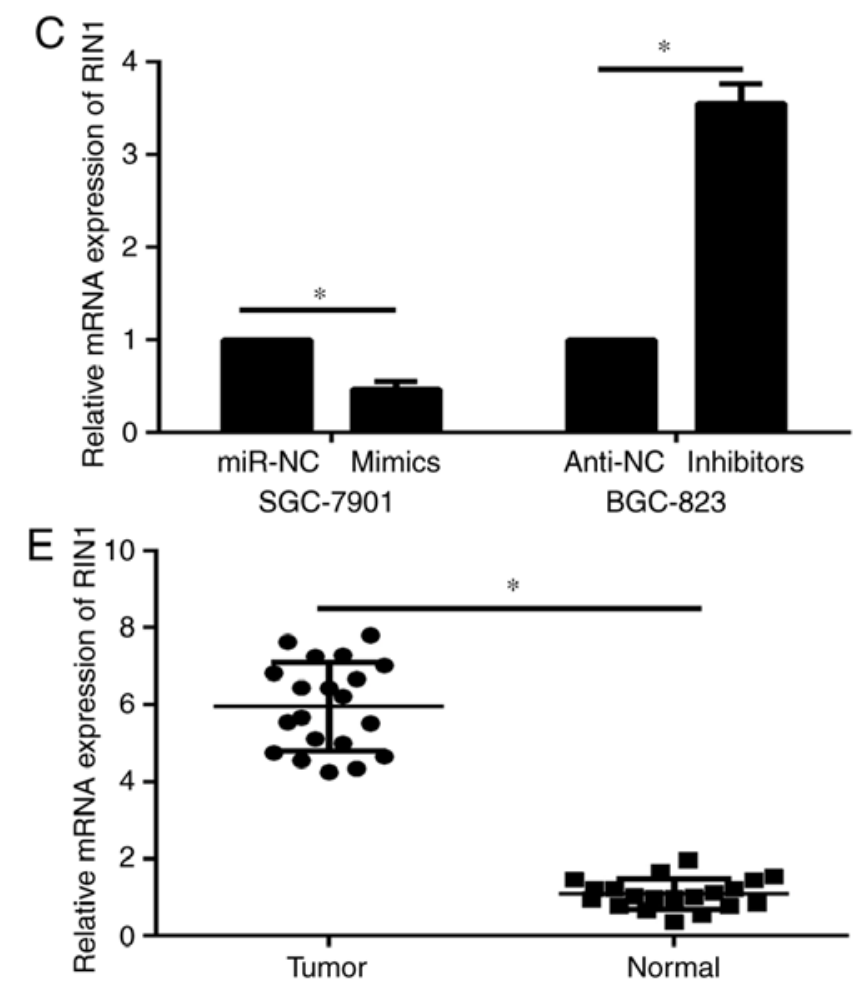

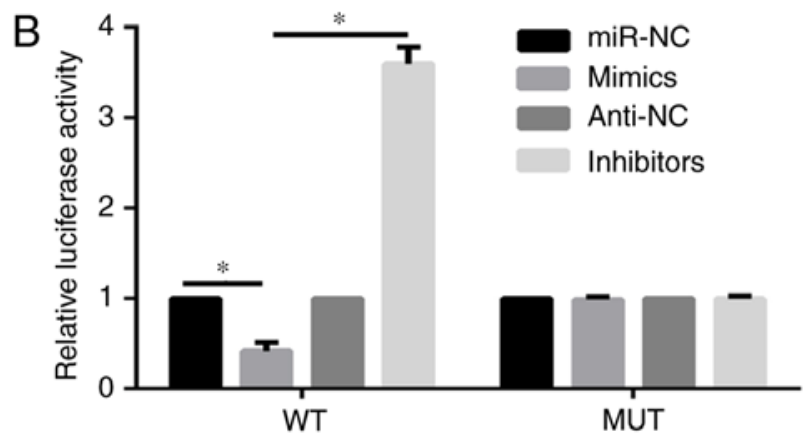

D
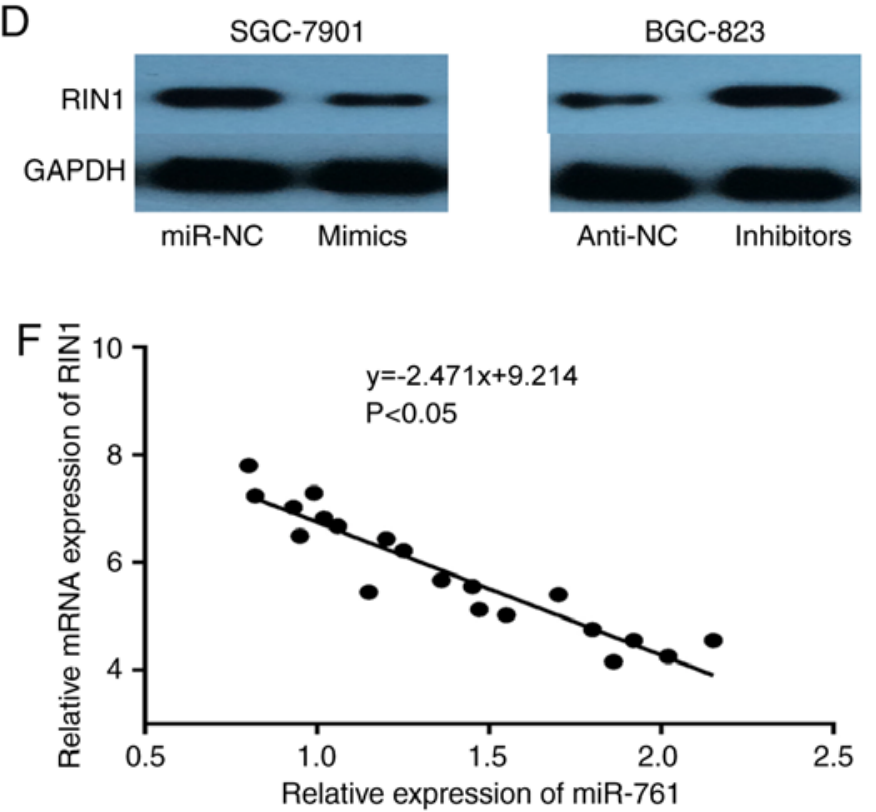

Figure 4. RIN1 is a direct downstream target of miR-761 in GC cells. (A) TargetScan prediction of miR-761 binding site on the 3'-UTR region of RIN1. (B) A dual-luciferase assay was performed on 293T cells co-transfected with the RIN1-3'-UTR-WT reporter plasmids and either miR-761 mimic or inhibitor. (C) A reverse transcription-quantitative PCR assay was used to analyze the RIN1 mRNA levels in response to miR-761 expression changes in GC cells. GAPDH acted as the internal control. (D) Western blot analysis was used to detect the RIN1 protein expression in response to miR-761 expression changes in GC cells. GAPDH acted as the loading control. (E) The mRNA expression level of RIN1 was analyzed in GC and adjacent normal gastric tissues. GAPDH acted as the normalization control. (F) Pearson's correlation analysis revealed the correlation between the expression levels of miR-761 and RIN1 mRNA in GC tissues. "P<0.05. miR, microRNA; 3'-UTR, 3'untranslated region; RIN1, Ras and Rab interactor 1; WT, wild-type; MUT, mutant; GC, gastric cancer; miR-NC, microRNA negative control; anti-NC, inhibitor negative control.

decreased RIN1 expression in miR-761-overexpression cells, and enhanced RIN1 expression in miR-761-silenced cells $(\mathrm{P}<0.05$; Fig. $4 \mathrm{C}, \mathrm{D})$. In addition, the mRNA expression of RIN1 in tumor tissues was significantly elevated compared with that in normal tissues $(\mathrm{P}<0.05$; Fig. $4 \mathrm{E})$, and a negative correlation between RIN1 mRNA and miR-761 expression was revealed in GC tissues $(\mathrm{P}<0.05$; Fig. $4 \mathrm{~F})$. These data suggest that miR-761 directly targets and regulates RIN1 expression in GC.

RIN1 is a functional downstream target of miR-761 in GC cells. This study investigated whether RIN1 overexpression was able to rescue the antitumor activity of miR-761 mimic in GC cells. SGC-7901 cells were transfected with miR-761 mimic and RIN1 overexpression plasmid. The western blotting results illustrated that the expression of RIN1 was enhanced in the RIN1 + miR-761 mimic group compared with that in the empty vector + miR-761 mimic group (Fig. 5A). The results of the CCK-8 and Transwell assays showed that RIN1 overexpression reversed the suppressive effects induced by the miR-761 mimic on GC cell proliferation $(\mathrm{P}<0.05$; Fig. $5 \mathrm{~B})$, migration and invasion $(\mathrm{P}<0.05$; Fig. $5 \mathrm{C}$ and $\mathrm{D})$. These data indicate that miR-761 may inhibit GC progression partially through the downregulation of RIN1.

\section{Discussion}

Although metastasis is the predominant cause of cancer-associated mortality, a comprehensive review of the modular and cellular determinants governing the related processes has not been conducted. Accumulating evidence has shown that miRNAs are aberrant in various types of cancer, and closely associated with progression and metastasis. Recent studies revealed that miR-761 presents important functions in human cancer types: It functions as an oncogene in breast cancer, hepatocellular carcinoma and non-small cell lung cancer $(11,12,14)$, and serves as a tumor suppressor in glioma, colorectal cancer and ovarian cancer $(9,10,13)$. However, the 

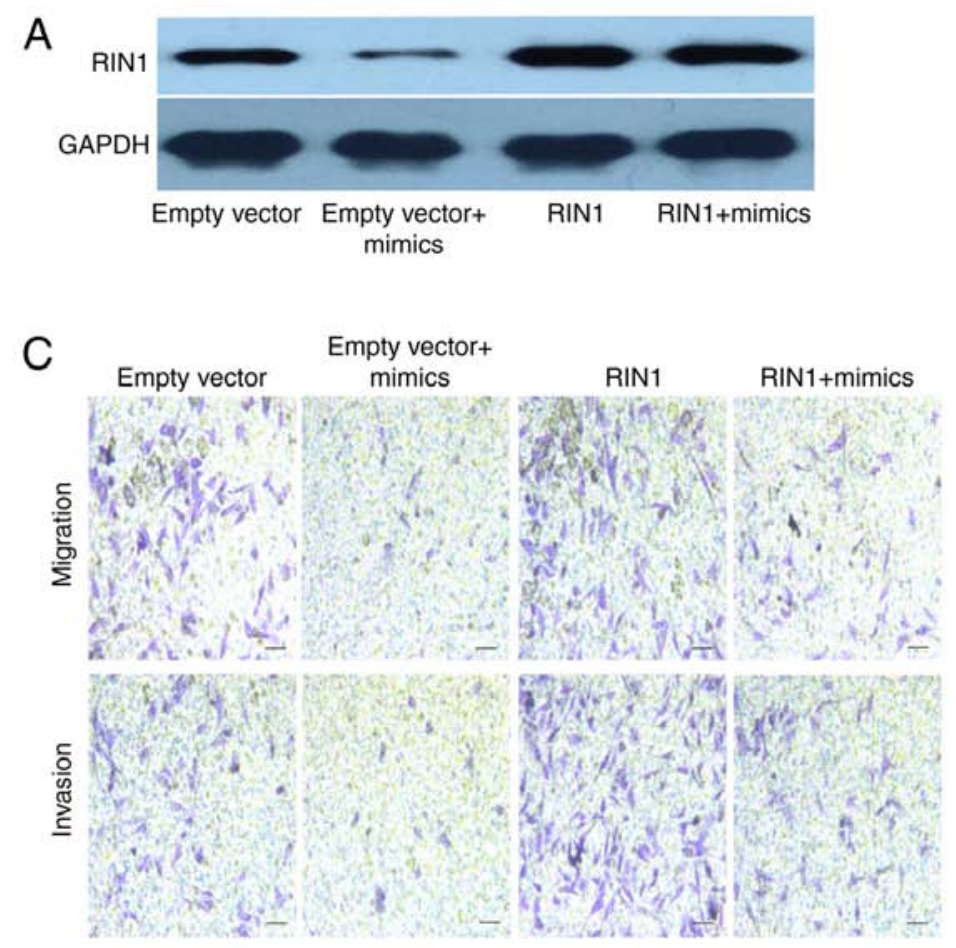
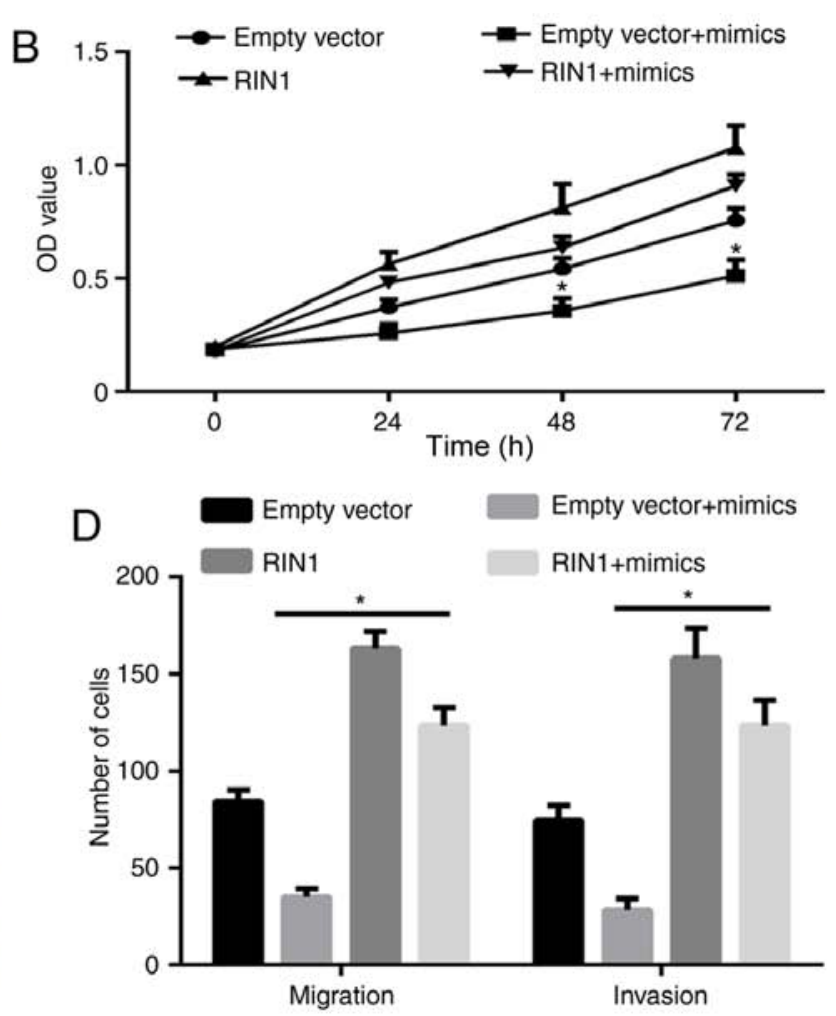

Figure 5. RIN1 is a functional target gene of miR-761 in gastric cancer cells. (A) The protein expression of RIN1 in SGC-7901 cells transfected with miR-761 mimic alone or miR-761 mimic + RIN1 overexpression plasmid. (B) The proliferation of SGC-7901 cells was analyzed using the Cell Counting Kit-8 assay. ${ }^{*} \mathrm{P}<0.05$ vs. miR-761 mimic + RIN1 overexpression plasmid. (C and D) The migration and invasion of SGC-7901 cells was detected using a Transwell assay. Scale bar, $100 \mu \mathrm{m}$. "P<0.05 empty vector + mimic group vs. miR-761 mimic + RIN1 overexpression plasmid. RIN1, Ras and Rab interactor 1 ; miR, microRNA.

expression, function and underlying molecular mechanism of miR-761 in GC have not been evaluated.

In the present study, miR-761 was found to be downregulated in GC tissues and cell lines, consistent with data from cancer types in which miR-761 acts as a tumor suppressor $(9,10,13)$. However, the association between miR-761 and the differentiation of GC cells was not investigated in this study. The functional assays demonstrated that miR-761 suppressed cell proliferation, migration and invasion. These data suggest that miR-761 serves as a tumor suppressor in GC.

Each miRNA targets certain genes, and the identification of these targets may help illustrate the pathogenesis of cancer (7). In the present study, the potential target of miR-761 was predicted using bioinformatics analysis, and RIN1 (a Ras effector protein) was identified as a molecule of interest. Previous studies have shown that RIN1 promotes tumor progression in non-small cell lung cancer, bladder urothelial carcinoma, renal cell carcinoma, melanoma, gastric adenocarcinoma and hepatocellular cancer (16-22). However, the mechanism of RIN1 in promoting tumor progression had not been determined. In this study, the relative expression of RIN1 in GC tissues was revealed, and RIN1 was significantly overexpressed in GC samples compared with normal tissues. It was also demonstrated that RIN1 is a direct target gene of miR-761, using a luciferase assay, RT-qPCR and western blotting. Furthermore, a negative correlation was revealed between RIN1 mRNA and miR-761 expression in GC tissues. Finally, the restoration of RIN1 was found to rescue the inhibition of proliferation, migration and invasion induced by the miR-761 mimic. These data therefore suggest that miR-761 suppresses GC progression by directly targeting RIN1. However, the signaling pathway regulated by the miR-761/RIN1 axis has not been explored.

A previous study reported that RIN1 regulated the epidermal growth factor receptor (EGFR) signaling pathway and promoted renal cell carcinoma malignancy in renal carcinogenesis (15). The association between miR-761 and the EGFR signaling pathway has not been illustrated, and this topic may be a novel research direction. In addition, the present study described the function and mechanism of miR-761 only in vitro. In future studies, in vivo experiments will be performed to support the present results. Thirdly, it is worth mentioning that each miRNA can regulate numerous genes, and multiple miRNAs may regulate the same gene. Therefore, the possibility of other target genes that may also be involved in the suppressive effects of miR-761 cannot be excluded, highlighting a further novel research direction.

In summary, this study revealed for the first time that miR-761 was significantly decreased in GC tissues and cell lines compared with normal tissues. miR-761 may serve as a tumor suppressor and decrease cell proliferation, migration and invasion by repressing RIN1 in GC cells. Therefore, miR-761 may be a potential biomarker for the prognosis of GC and a novel therapeutic target for treating advanced stages of this disease. 


\section{Acknowledgements}

Not applicable.

\section{Funding}

No funding was received.

\section{Availability of data and materials}

The datasets used and/or analyzed during the present study are available from the corresponding author on reasonable request.

\section{Authors' contributions}

QZ and XS conceived and designed the experiments. QZ, YS and XS conducted all of the experiments. YS and XS wrote and revised the manuscript. All authors read and approved the final manuscript.

\section{Ethics approval and consent to participate}

The present study was approved by the ethics committee of the Affiliated Hospital of Weifang Medical University (approval no. WYFY20150109002). Prior written informed consent was obtained from each patient.

\section{Patient consent for publication}

Not applicable.

\section{Competing interests}

The authors declare that they have no competing interests.

\section{References}

1. Van Cutsem E, Sagaert X, Topal B, Haustermans K and Prenen H: Gastric cancer. Lancet 388: 2654-2664, 2016.

2. Choi IJ, Lee JH, Kim YI, Kim CG, Cho SJ, Lee JY, Ryu KW, Nam BH, Kook MC and Kim YW: Long-term outcome comparison of endoscopic resection and surgery in early gastric cancer meeting the absolute indication for endoscopic resection. Gastrointest Endosc 81: 333-341 e1, 2015.

3. Fukagawa T, Katai H, Mizusawa J, Nakamura K, Sano T, Terashima M, Ito S, Yoshikawa T, Fukushima N, Kawachi Y, et al: A prospective multi-institutional validity study to evaluate the accuracy of clinical diagnosis of pathological stage III gastric cancer (JCOG1302A). Gastric Cancer 21: 68-73, 2018.

4. Smyth EC, Verheij M, Allum W, Cunningham D, Cervantes A and Arnold D; ESMO Guidelines Committee: Gastric cancer: ESMO Clinical Practice Guidelines for diagnosis, treatment and follow-up. Ann Oncol 27 (Suppl 5): v38-v49, 2016.

5. Valinezhad Orang A, Safaralizadeh R and Kazemzadeh-Bavili M Mechanisms of miRNA-mediated gene regulation from common downregulation to mRNA-specific upregulation. Int J Genomics 2014: 970607, 2014.
6. Macfarlane LA and Murphy PR: MicroRNA: Biogenesis, function and role in cancer. Curr Genomics 11: 537-561, 2010.

7. Yuan HL, Wang T and Zhang KH: MicroRNAs as potential biomarkers for diagnosis, therapy and prognosis of gastric cancer. Onco Targets Ther 11: 3891-3900, 2018.

8. Dehghanzadeh R, Jadidi-Niaragh F, Gharibi T and Yousefi M: MicroRNA-induced drug resistance in gastric cancer. Biomed Pharmacother 74: 191-199, 2015.

9. Li GF, Li L, Yao ZQ and Zhuang SJ: Hsa_circ_0007534/ miR-761/ZIC5 regulatory loop modulates the proliferation and migration of glioma cells. Biochem Biophys Res Commun 499: 765-771, 2018.

10. Cao S, Lin L, Xia X and Wu H: MicroRNA-761 promotes the sensitivity of colorectal cancer cells to 5-Fluorouracil through targeting FOXM1. Oncotarget 9: 321-331, 2017.

11. Guo GC, Wang JX, Han ML, Zhang LP and Li L: microRNA-761 induces aggressive phenotypes in triple-negative breast cancer cells by repressing TRIM29 expression. Cell Oncol (Dordr) 40: 157-166, 2017.

12. Zhou X, Zhang L, Zheng B, Yan Y, Zhang Y, Xie H, Zhou L, Zheng S and Wang W: MicroRNA-761 is upregulated in hepatocellular carcinoma and regulates tumorigenesis by targeting Mitofusin-2. Cancer Sci 107: 424-432, 2016.

13. Shi $\mathrm{C}$ and Zhang $\mathrm{Z}$ : miR-761 inhibits tumor progression by targeting MSI1 in ovarian carcinoma. Tumour Biol 37: 5437-5443, 2016

14. Yan A, Yang C, Chen Z, Li C and Cai L: MiR-761 promotes progression and metastasis of non-small cell lung cancer by targeting ING4 and TIMP2. Cell Physiol Biochem 37: 55-66, 2015.

15. Livak KJ and Schmittgen TD: Analysis of relative gene expression data using real-time quantitative PCR and the 2(-Delta Delta $\mathrm{C}(\mathrm{T})$ ) method. Methods 25: 402-408, 2001.

16. Feng ZH, Fang Y, Zhao LY, Lu J, Wang YQ, Chen ZH, Huang Y, Wei JH, Liang YP, Cen JJ, et al: RIN1 promotes renal cell carcinoma malignancy by activating EGFR signaling through Rab25. Cancer Sci 108: 1620-1627, 2017

17. Li F, Fang Z, Zhang J, Li C, Liu H, Xia J, Zhu H, Guo C, Qin Z, $\mathrm{Li}$ F, et al: Identification of TRA2B-DNAH5 fusion as a novel oncogenic driver in human lung squamous cell carcinoma. Cell Res 26: 1149-1164, 2016.

18. Liu HY, Wu G, He H, Wang YW, Cheng Y and Liu YF: RIN1 expression in hepatocellular cancer and the affection on prognosis and tumor invasion ability. Zhonghua Wai Ke Za Zhi 51: 1025-1029, 2013 (In Chinese).

19. Fang P, Zhao Z, Tian H and Zhang X: RIN1 exhibits oncogenic property to suppress apoptosis and its aberrant accumulation associates with poor prognosis in melanoma. Tumour Biol 33: $1511-1518,2012$.

20. Yu HF, Zhao G, Ge ZJ, Wang DR, Chen J, Zhang Y, Zha TZ, Zhang K, Zhang M, Tan YF, et al: High RIN1 expression is associated with poor prognosis in patients with gastric adenocarcinoma. Tumour Biol 33: 1557-1563, 2012.

21. Shan GY, Zhang Z, Chen QG, Yu XY, Liu GB and Kong CZ: Overexpression of RIN1 associates with tumor grade and progression in patients of bladder urothelial carcinoma. Tumour Biol 33: 847-855, 2012.

22. Wang Q, Gao Y, Tang Y, Ma L, Zhao M and Wang X: Prognostic significance of RIN1 gene expression in human non-small cell lung cancer. Acta Histochem 114: 463-468, 2012.

This work is licensed under a Creative Commons Attribution-NonCommercial-NoDerivatives 4.0 International (CC BY-NC-ND 4.0) License. 